\title{
Multi-frequency observations of radio enhanced temperature regions of the Sun
}

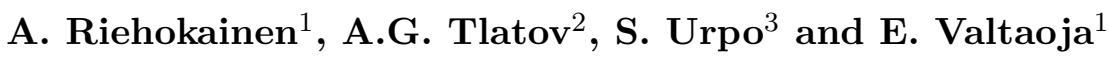 \\ ${ }^{1}$ Tuorla Observatory, Turku University, Finland email: alerie@utu.fi \\ ${ }^{2}$ Kislovodsk Solar Station of the Pulkovo Observatory, Russia, ${ }^{3}$ Metsahovi Radio Observatory, \\ Helsinki Univ. of Technology, Finland
}

\begin{abstract}
We compare the enhanced temperature regions (ETRs) in the millimeter radio emission of the Sun with other manifestation of solar activity during 10 days in 1997, 1998, 2000 and 2003. The radio observations at $37 \mathrm{GHz}$ and $87 \mathrm{GHz}$ were done with the Metsahovi Radio Telescope (Finland). We concentrate on the weak ETRs with no discernible connection to sunspots, and in some case compare their properties with those which do have a clear connection to sunspots. Extended observations at $37 \mathrm{GHz}$ in July 2003 enable us to measure the lifetimes of some weak ETRs and also their brightness temperature variations. The $37 / 87 \mathrm{GHz}$ full-disk radio intensity contours are superposed on $C a I I\left(k_{3}\right)$ and $H_{\alpha}$ images observed with the Meudon Spectroheliograph (France) for comparing the optical positions and the brightness characteristics of these regions. A similar comparison is also made with the SOHO/MDI fulldisk magnetograms and SOHO/EIT images. There appear to be two different classes of weak ETRs. One class is connected with local brightness features seen in $\operatorname{CaII}\left(k_{3}\right), H_{\alpha}$ images and magnetograms. The other class is connected with so-called minor brightness features.
\end{abstract}

Enhanced temperature regions (ETRs) are the object of our investigations during some years. Initially we used the ETRs abbreviation only for a special class of objects, namely for the radio brightenings which were discovered in the polar coronal holes by Babin et al. (1976). It was surprise that coronal holes appear bright in $1 \mathrm{~cm}$ radio observations. Also, there are ETRs at low solar latitudes, the larger part of which can be associated with faculae. Equatorial coronal holes are also enhanced, but they have been associated with $H_{\alpha}$ intranetwork brightenings by Moran et al. (2001). In our previous work (Riehokainen et al. (2003)) we showed that weak ETRs at $87 \mathrm{Ghz}$ and $37 \mathrm{GHz}$ are connected to some brightness structures. In some cases these structures look like cels; in other cases these ETRs were connected with the local brightest and diffuse brightness structures. Special investigations carried out by various authors have suggested that ETRs could be connected with magnetic field configurations (Gopalswamy et al. (1999)), diffuse brightenings, bright points and polar plumes (Pohjolainen et al. (2000)) and polar faculae (Riehokainen et al. (1998)). However, no one-to-one correlations with the abovementioned features has been found. This led us to assume that there are at least two different optical brightness structures, which produce apparantly similar radio ETRs. One goal of this study was to test this assumption. Some important characteristics, such as lifetimes and brightness variations of ETRs have not been investigated very well previously. Another goal of this work to study the radio variations of ETRs.

Figure 1 show variations of the radio brightness for some different ETRs. The latitude and longitude range of each ETR are given in the bracets. The brightness of the low latitudes ETRs remains approximately constant, except for the ETR 15-1, which is connected with sunspot and shows a two hour burst. The brightness of the polar coronal hole ETRs are smaller, with lifetimes from tens of minutes to a few hours. 


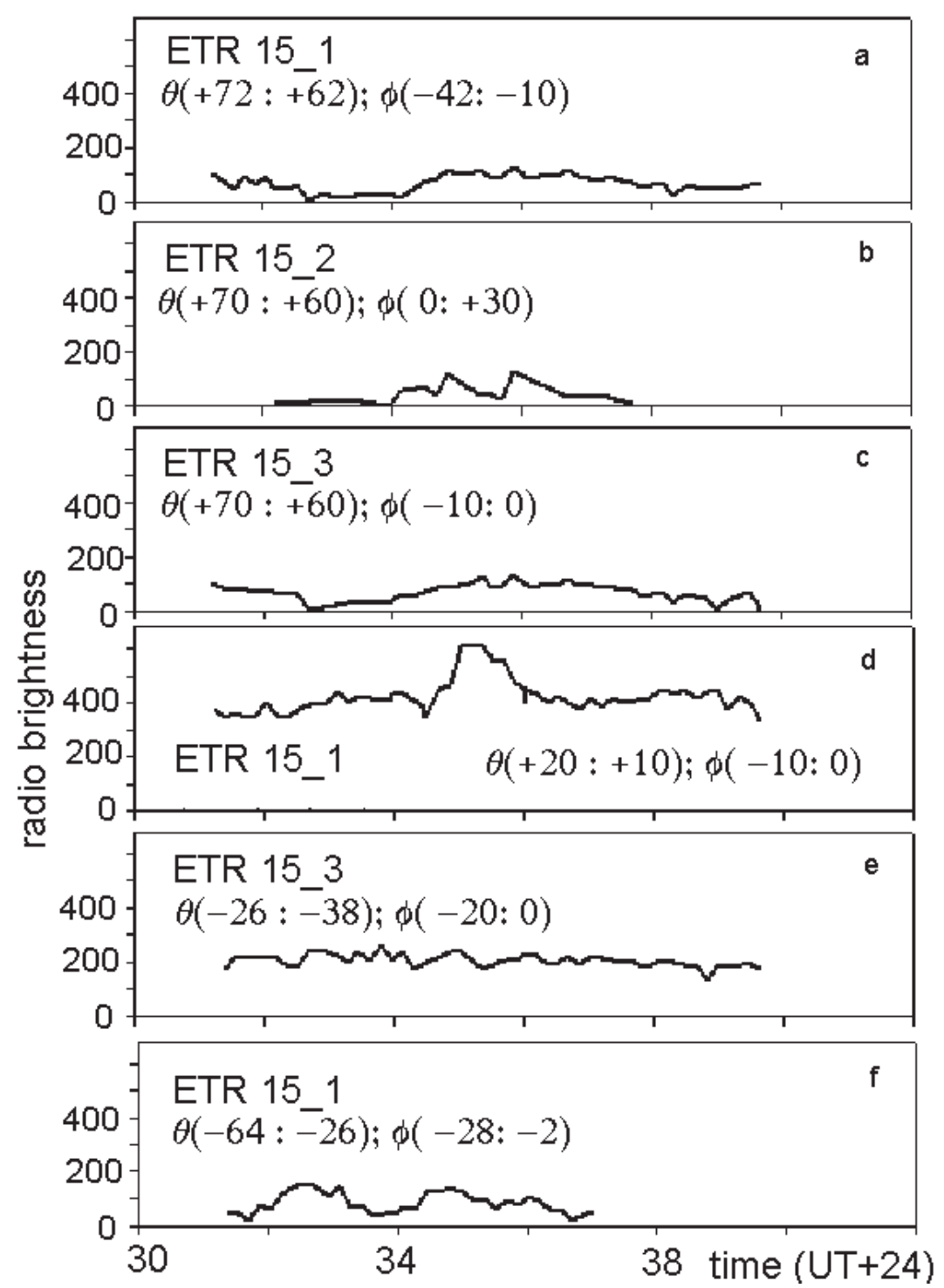

Figure 1. Temporal variation of radio brightness for the ETRs on 15.07.2003

ETRs (on the north) situated relatively close to each other seem to show some synchronization in their brightness variations. However, this effect needs futher investigation.

\section{References}

Babin, A.N., Gopasyuk, S.I., \& Efanov, V.A., 1976 Izv. Krymsk. Astrophys. Obs.55

Gopalswamy, N., Shibasaki, K., Thompson., Gurman, J., \& DeForest, C., 1999 JGR p.104

Moran, T., Gopalswamy, N., Dammash, I.E. \& Wilhelm, K., 2001 A $\& A$ 378, p.1037

Pohjolainen, S., Portier-Fzzani, F., \& Ragaigne, D., 2000 A $\& A$ 143, p.227

Riehokainen, A., Urpo, S., Valtaoja, E., 1998 A $\& A$ 333, p.741

Riehokainen, A., Valtaoja, E., Pohjolainen, S., 2003 A\&A 402, p.1103 